\title{
Aa. Vv., Hantises, «Ponts/Ponti»
}

\section{Emanuela Cacchioli}

\section{(2) OpenEdition}

\section{Journals}

\section{Edizione digitale}

URL: http://journals.openedition.org/studifrancesi/5795

DOI: 10.4000/studifrancesi.5795

ISSN: 2421-5856

\section{Editore}

Rosenberg \& Sellier

\section{Edizione cartacea}

Data di pubblicazione: 1 septembre 2011

Paginazione: 467-468

ISSN: 0039-2944

\section{Notizia bibliografica digitale}

Emanuela Cacchioli, «Aa. Vv., Hantises, «Ponts/Ponti»», Studi Francesi [Online], 164 (LV | II) | 2011, online dal 30 novembre 2015, consultato il 09 janvier 2021. URL: http://journals.openedition.org/ studifrancesi/5795 ; DOI: https://doi.org/10.4000/studifrancesi.5795

Questo documento è stato generato automaticamente il 9 janvier 2021.

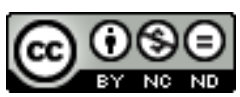

Studi Francesi è distribuita con Licenza Creative Commons Attribuzione - Non commerciale - Non opere derivate 4.0 Internazionale. 


\title{
Aa. Vv., Hantises, «Ponts/Ponti»
}

\author{
Emanuela Cacchioli
}

\section{NOTIZIA}

Hantises, «Ponts/Ponti», n. 10, 2010, 303 pp.

1 L'ultimo numero della rivista è dedicato al tema dell'ossessione e alla sua insistente presenza nelle opere francofone. La prima sezione raccoglie quattro saggi che analizzano l'argomento nella letteratura belga, africana e caraibica. In particolare, Marc QUAGHEBEUR con Carnevalesque et crépusculaire, un Ulysse de la fin de l'Histoire. "La Mélancolie du voyeur» de et chez Conrad Detrez (pp. 13-38) conduce uno studio sull'opera postuma e incompiuta dell'autore belga, naturalizzato francese. L'articolo indaga il percorso biografico e letterario di Detrez, attraverso il testo che si presenta come una sorta di autobiografia romanzata, in cui autore, narratore e figure fittizie si sovrappongono per esprimere la malinconia che colpisce Detrez come individuo e membro di una collettività (i belgi francofoni, infatti, devono confrontarsi con la percezione di una comunanza con la Francia dettata dall'elemento linguistico, che si scontra, tuttavia, con un'ingerenza a livello politico e amministrativo da parte dei Paesi Bassi). L'ossessione di Detrez si concretizza con l'immagine della Storia che, a sua volta, si coniuga con l'Identità e l'Alterità. Una Storia che coinvolge soggetti ed epoche, li fa diventare protagonisti (lo stesso Detrez ha partecipato alla deposizione del regime dittatoriale di Branco in Brasile) e, infine, riduce se stessa a spettro. L'uomo diventa, così, un osservatore distaccato che può avvalersi soltanto della memoria: si tratta dell'ultima attività concessa ad un soggetto malato e consapevole che la sua vita è giunta al termine. Eppure il narratore riesce a mantenere uno sguardo ironico e uno stile barocco carnevalesco a cui si aggiungono momenti di epifanie fantastiche che non fanno mai perdere di vista l'obiettivo del suo viaggio di novello Ulisse: la ricerca della Bellezza. Interroger la Lettre, ou la Hantise logogriphe dans "Passage des larmes" de Abdourahaman A. Waberi (pp. 39-64) è il titolo del saggio di Silvia RIVA, che mette in luce come nel romanzo dell'autore gibutiano l'esilio, il nomadismo e i paesaggi mentali possano raggiungere una forma ossessiva molto complessa che si realizza nella 
scrittura. Waberi, sfruttando la struttura di un thriller cinematografico, narra la vicenda di un connazionale che, tornato a Gibuti per lavoro, è costretto a confrontarsi con il proprio passato e un fratello gemello che prova rancore verso di lui. I due personaggi hanno la funzione di duplicare la narrazione e di instaurare un intreccio di due vicende che si alternano, convergono e creano una rete di scambi. La scrittura acuisce ancora di più tali relazioni, in quanto il fratello che rientra a Gibuti ha il compito di trascrivere su un taccuino la situazione sociale per favorire eventuali commerci da parte degli americani, mentre l'altro gemello, incarcerato, segna su fogli di fortuna le indicazioni che una guida fondamentalista vuole insegnargli. Il risultato è che il primo fratello lascia che favole, leggende e proverbi della tradizione orale trovino un supporto scritto, mentre il secondo scopre che un compagno di cella ha annotato su uno dei fogli che sta utilizzando un messaggio di Walter Benjamin che merita di essere tramandato ai posteri. Sarà proprio tale missione che darà un senso alla sua vita. La scrittura, dunque, viene percepita come nomadismo, inteso non come condizione fisica, bensì mentale. In un mondo votato al decentramento, esistono frammenti di scrittura sparsi in epoche e aree geografiche diverse. Occorre, dunque, individuare una guida che ci indichi il percorso per formulare un messaggio di senso compiuto. Waberi ci suggerisce di rintracciarla nello stesso Benjamin. Nel saggio Retour au pays natal, espoir ou hantise chez les écrivains haïtiens de la diaspora (pp.65-82), Alba PESSINI si focalizza, invece, sulla situazione haitiana. Per molti scrittori rimasti a lungo lontano da Haiti, il ritorno nel paese natale è un'ossessione da intendersi come pensiero ricorrente e costante, ma anche come timore di ritrovare un luogo profondamente cambiato. Il rientro sull'isola, analizzato nelle varianti di "ritorno problematico" (in quanto si crea uno sfasamento tra il paese attuale e quello rimasto nella memoria) e di "ritorno denunciatore" (che si concretizza con una vera e propria inchiesta da parte dell'autore), è il nucleo della riflessione di Émile Ollivier, Anthony Phelps e Dany Laferrière. Sebbene ognuno di questi scrittori indaghi l'esperienza del ritorno in modo personale, si possono rintracciare delle costanti che accomunano i loro testi. Innanzitutto abbiamo a che fare con un rientro enigmatico in cui si crea uno scarto tra il paese presente nella memoria dell'autore e quello che appare concretamente ai suoi occhi. Una distanza che si manifesta attraverso la perdita dell'intimità con la terra natale e che nemmeno la riappropriazione fisica riesce a colmare. L'unico modo per ritrovare il paesaggio mentale è quello di ricorrere alla memoria e alla conseguente trasfigurazione in un mondo onirico che si inserisce in una dimensione temporale altra. E la scrittura, in questo caso, permette di rivivere i momenti del passato legati a tale paesaggio ora epurato degli aspetti negativi. Nel quarto saggio, dal titolo Hantise du présent, hantise du passé dans «Les Derniers rois mages» de Maryse Condé (pp. 83-102), Simonetta VALENTI spiega come la scrittrice guadalupana abbia preferito assumere l'ossessione del presente in tutte le sue contraddizioni piuttosto che risalire ad un passato ormai vuoto di significato. Spéro, il protagonista del romanzo, si è trasferito negli Stati Uniti per sfuggire alla situazione di povertà in cui versa il suo paese e per ottenere la fama per la sua attività artistica. Dopo un'iniziale fase di successo, conosce il fallimento a livello professionale (i suoi quadri non sono più apprezzati) e nella vita matrimoniale. Nell'ultima fase della sua esistenza, inoltre, Spéro si rende conto che rintracciare il legame che unisce i Caraibi con le tradizioni africane è ormai divenuto impossibile in quanto gli occidentali hanno esportato il loro modello anche in Africa e ciò ha determinato uno scarto rispetto alle radici che hanno segnato la nascita della Guadalupa. Non restano che una serie di riti e di concezioni mitiche ormai svuotate di 
significato. La rottura con il passato è il passaggio fondamentale per accettare la ricchezza e l'originalità del patrimonio creolo e metterlo in dialogo con altre culture a partire dalla diversità che le caratterizza. Segue poi un articolo dedicato agli studi linguistici. Gabriella VERNETTO, come ci indica il suo intervento, Les épreuves de français de fin de cycle en contexte bi/plurilingue: le cas de la Vallée d'Aoste (pp. 105-124) si concentra sulla questione della valutazione del francese nel contesto geografico della Val d'Aosta, regione in cui è lingua ufficiale. Oltre ad analizzare i metodi di somministrazione e di correzione dei test, il saggio si focalizza su eventuali proposte alternative di valutazione del livello linguistico acquisito dagli studenti anche in relazione all'introduzione di uno strumento quale il Portfolio. Il volume è, inoltre, corredato da una serie di ampie recensioni relative a studi linguistici e letterari sulla francofonia europea e mondiale. 